\title{
A New Preparation Process of Double-gate FETs Based on Monolayer Graphene Nanoflakes
}

\author{
Zong-Qi BAI 1,a , Ka-Di ZHU ${ }^{1, b, *}$,Hang YANG ${ }^{2, c}$ and Xue-Ao ZHANG ${ }^{2, d}$ \\ ${ }^{1}$ Department of Physics and Astronomy, Shanghai Jiaotong University, Shanghai 200240,China \\ ${ }^{2}$ College of Science, National University of Defense Technology, Changsha 410073, China

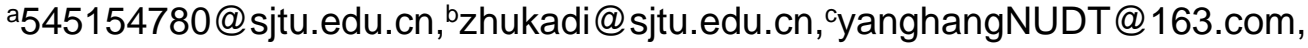 \\ dxazhang@nudt.edu.cn, \\ ${ }^{*}$ Corresponding author
} Keywords: Monolayer graphene nanoflakes, Double-gate field effect transistor (DG-FET),
Characterization of DG-FET

\begin{abstract}
A new preparation process to product double-gate field effect transistor (DG-FET) of monolayer graphene nanoflakes, fabricated by mechanical exfoliation, was systematically studied in this paper. The graphene DG-FETs are bipolar and possess high gate modulation (On/Off ratio is large), and higher regulation accuracy, which is double-gate regulation. These results showed the extraordinary performance of the double-gate FETs based on monolayer graphene, which might open a new road to develop graphene-based material in the application of double-gate FETs.
\end{abstract}

\section{Introduction}

Since graphene has been discovered by Geim and Novoselov in 2004[1], this two-dimensional (2D) material with typically extraordinary properties has attracted extensive research in the last few years[2-5]. Disappointingly, low On/Off ratio and low regulation accuracy has immensely restricted the application of this star material in logic transistor field and optoelectronics[6,7]. Fortunately, DG-FET is an effective way to solve the problem, which has extraordinary properties. So it is necessary to expand a better path to produce the DG-FET.

Although the concept of DG-FET has been put forward for a long time[9-16], few researchers can stably produce DG-FET. Therefore, a new preparation process of DG-FET based on monolayer graphene is systematically put forward in this paper, which exhibits extraordinary properties[16-20].

Results indicate that the graphene DG-FET, fabricated by the new way, has a larger On/Off ratio and higher regulation accuracy, compared to traditional graphene FETs and graphene DG-FETs are produced by other way. More importantly, we open up a new road to produce monolayer graphene DG-FET stably and normatively. Accordingly, this graphene DG-FET may possibly be more promising in the application of FETs.

\section{Experimental Details}

Device fabrication: The DG-FETs based on graphene were fabricated by standard micro-nano technology(EBL, ALD and EBE). The preparation is divided into two parts: back gate preparation and top gate preparation. 

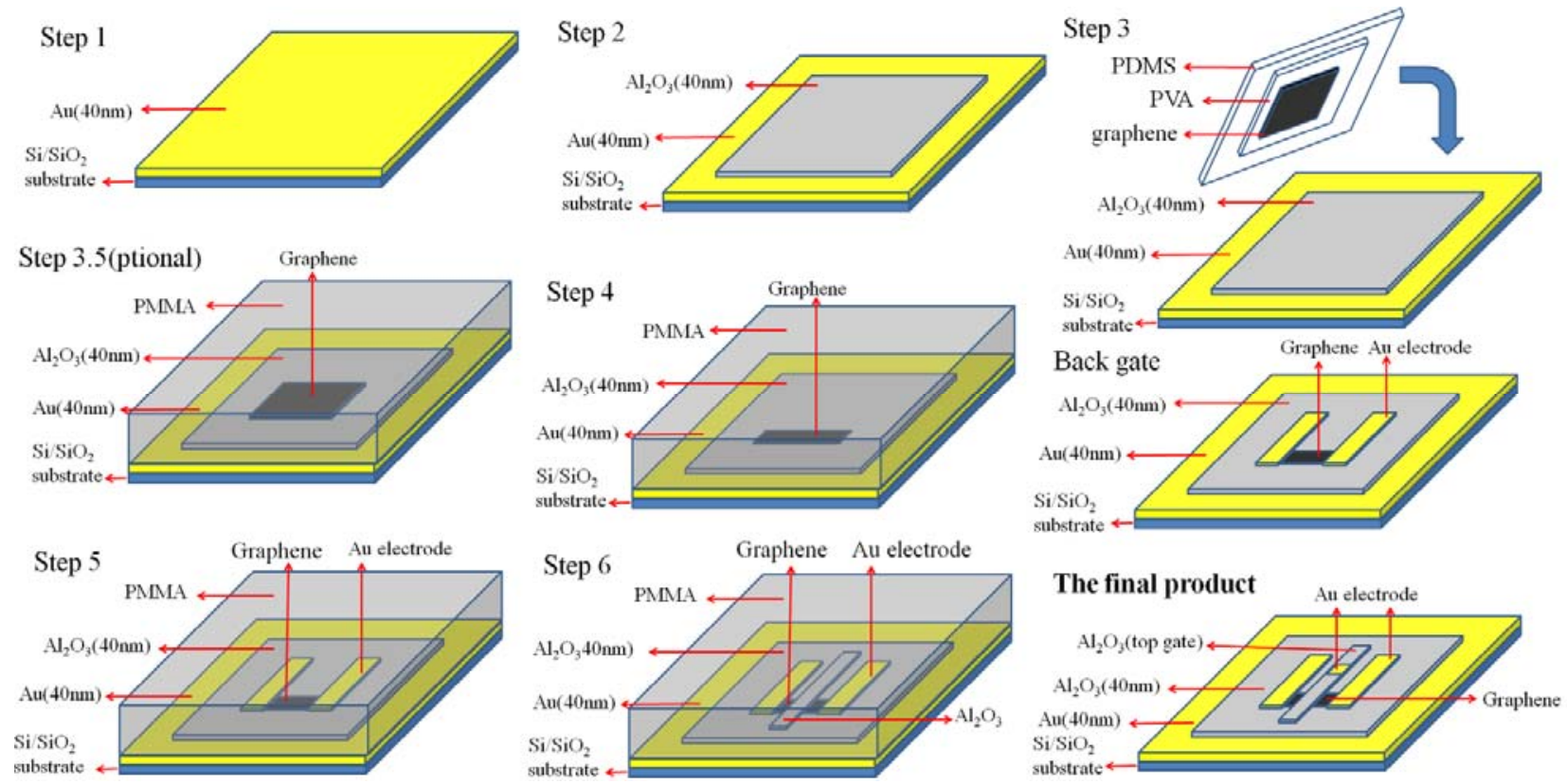

Figure 1. steps of the preparation of DG-FETs which is divided into two parts. (a) four steps of the preparation of back gate.(b)the other two steps of the preparation of top gate and the final DG-FET.

Firstly, The gold is evaporated onto $300 \mathrm{~nm} \mathrm{Si} / \mathrm{SiO}_{2}$ (doped p++, conductivity: $0.01-0.02 \Omega \cdot \mathrm{cm}$ ) substrate About $40 \mathrm{~nm}$ which fabricated by EBL(electron beam lithography) and EBE(electron beam evaporation). Secondly, $5 \mathrm{~nm}$ of alumina $\left(\mathrm{Al}_{2} \mathrm{O}_{3}\right)$ was deposited on the $\mathrm{Au}$ film by ALD(atomic layer deposition) with somewhere uncovered. Thirdly, the graphene nanoflakes were exfoliated from the graphite crystals onto $300 \mathrm{~nm} \mathrm{SiO} / / \mathrm{Si}$ substrates using mechanical exfoliation technique and find suitable monolayer graphene sample under the microscope. Therefore, The graphene sample was transferred onto the $\mathrm{Al}_{2} \mathrm{O}_{3}$ film by heterojunction fixed point transfering technique. There is a step optional that the monolayer graphene had better be etched away after covered PMMA and lithography if it is irregular in shape after step 3. Fourthly, gold electrode was made on graphene after several step: rotating rubber, EBL, EBE and acetone cleaning. Up to now, back gate preparation, half of all, is completed.

The other parts, preparation of top gate, are of vital importance. Fifthly, Making the shape of top gate by EBL in the sample which covered by PMMA, and covering the shape, fabricated by ALD System, by $\mathrm{Al}_{2} \mathrm{O}_{3}$. By the way, the $\mathrm{Al}_{2} \mathrm{O}_{3}$ should not cover the $\mathrm{Au}$ electrode. Finally, once again like step 4 to make Au electrode in top gate. Thus, we get the final product double-gate monolayer graphene field effect transistor.

\section{Results and Discussion}

Characterization of monolayer graphene nanoflakes and DG-FETs: Firstly, The DG-FETs were characterized by SEM to comprehend the surface photography of Alumina and section profile. Secondly, the graphene nanoflakes were characterized by Raman spectroscopy to comprehend the substance type. Finally, The graphene nanoflakes were characterized by SEM to comprehend the distance between the Au electrode. 

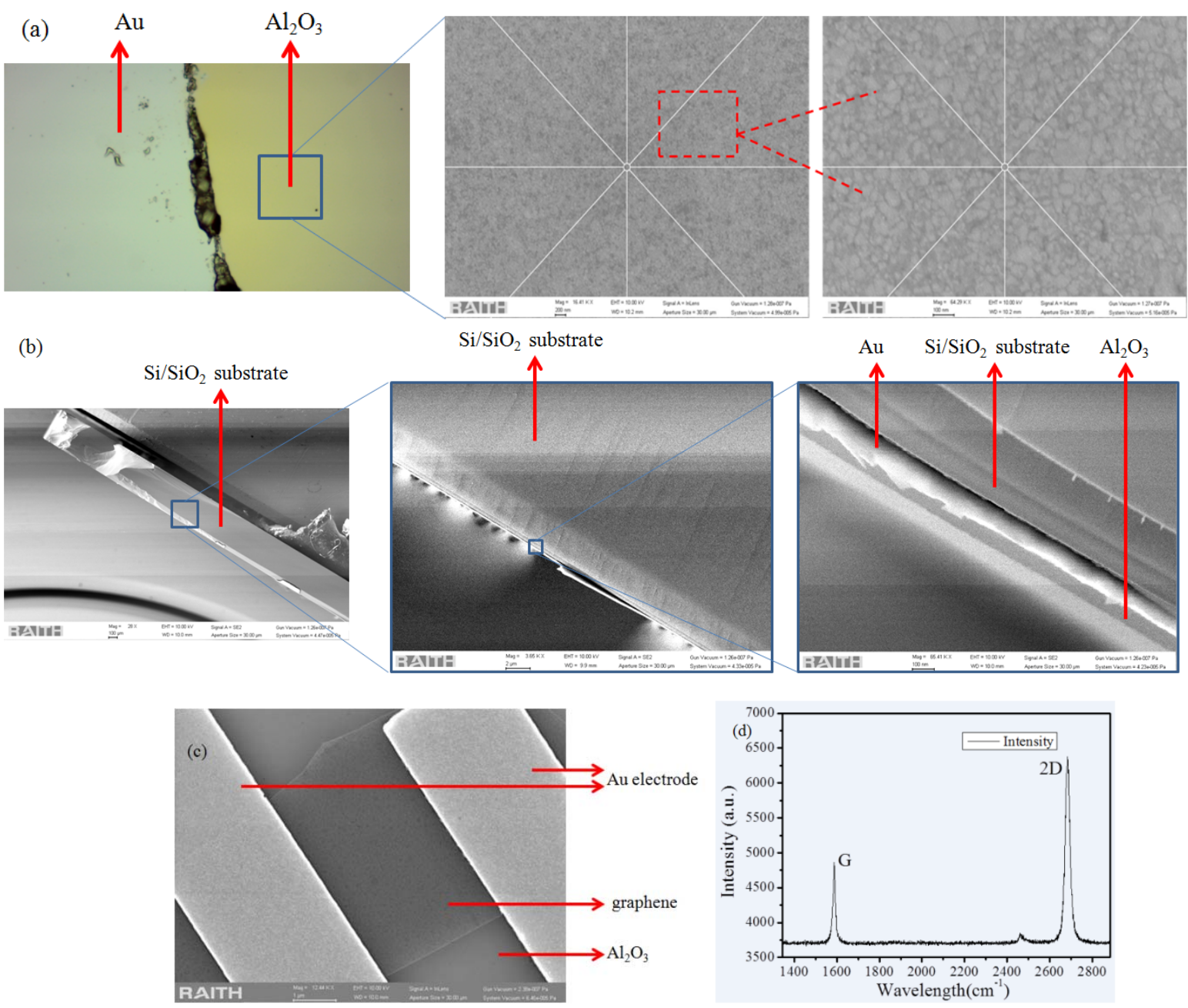

Figure 2. Characterization of monolayer graphene nanoflakes and FETs. (a) Photography of the interface of $\mathrm{Al}_{2} \mathrm{O}_{3}-\mathrm{Au}$ in the top view under optical microscope and its SEM image in $16.41 \mathrm{k} \mathrm{X}$ and 64.29k X magnification. (b) SEM image of the interface of $\mathrm{Al}_{2} \mathrm{O}_{3}-\mathrm{Au}-\mathrm{Si} / \mathrm{SiO}_{2}$ in the side view in 28

$\mathrm{X}, 3.65 \mathrm{k} \mathrm{X}$ and $65.41 \mathrm{k} X$ magnification. (c) SEM image of the back gate of DG-FET in the top view (h) Raman spectra of monolayer graphene.

Results of characterization: Firstly, figure 2(a) shows the photography of the interface of $\mathrm{Al}_{2} \mathrm{O}_{3}-\mathrm{Au}$ in the top view under optical microscope and its SEM image in $16.41 \mathrm{k} \mathrm{X}$ and $64.29 \mathrm{k} \mathrm{X}$ magnification and there is a clear boundary between the two regions. And the $\mathrm{Al}_{2} \mathrm{O}_{3}$ film is smooth and compact. Secondly, figure 2(b) show the SEM image of the interface of $\mathrm{Al}_{2} \mathrm{O}_{3}-\mathrm{Au}-\mathrm{Si} / \mathrm{SiO}_{2}$ in the side view in $28 \mathrm{X}, 3.65 \mathrm{k}$ X Thirdly, figure 2(c) shows the SEM image of the back gate of DGFET and the production of the back gate of DG-FET is successful. Finally, figure 2(d) shows the raman spectra of monolayer graphene and proved that it is indeed graphene.

\section{Summary}

To summary, we have fabricated DG-FETs based on monolayer graphene. Due to the unique structural properties of two-dimensional material, DG-FETs based on it exhibit a high gate modulation (On/Off ratio is large), and higher regulation accuracy. In our experiment, we fabricated DG-FETs by standard micro-nano technology and characterized it by SEM and Raman spectra. Obviously, we fabricated DG-FETs with great quality based on monolayer graphene successfully. And our next task is to study its electrical properties in depth. In all, the new preparation method to 
product double-gate field effect transistor, we believe, will open up a new way in the application of FETs.

\section{Acknowledgement}

This work was supported by the National Natural Science Foundation of China (No.11574395, No.11274230 and No.11574206), the Research Programme of National University of Defense Technology (No. JC15-02-01), the open foundation of State Key Laboratory of high Performance Computing (No. 201301-02), and the open foundation based on the innovation platform of Hunan key laboratories (No. 13K022).

\section{References}

[1] Novoselov K S, Geim A K, Morozov S V, et al. Electric Field Effect in Atomically Thin Carbon Films[J]. Science, 2004, 306(5696):666.

[2] Novoselov K S, Jiang Z, Zhang Y, et al. Room-temperature quantum Hall effect in graphene.[J]. Science, 2007, 315(5817):1379.

[3] Bertolazzi S, Krasnozhon D, Kis A. Nonvolatile memory cells based on MoS2/graphene heterostructures.[J]. Acs Nano, 2013, 7(4):3246-52.

[4] De Abajo F J G, Koppens F H L, Chang D E, et al. Graphene Plasmonics[C]// International Workshop on Theoretica. American Institute of Physics, 2011:28-29.

[5] Avouris $\mathrm{P}$, Lin $\mathrm{Y} \mathrm{M}$, Mueller $\mathrm{T}$, et al. Ultrafast graphene photodetector[J]. Nature Nanotechnology, 2012, 4(12):839-843.

[6] Geim A K, Novoselov K S. The rise of graphene.[J]. Nature Materials, 2007, 6(3):183-191.

[7] Geim A K. Science, Graphene: Status and Prospects[J]. Science, 2009, 324(5934):1530-1534.

[8] Hisamoto D, Lee W C, Kedzierski J, et al. FinFET-a self-aligned double-gate MOSFET scalable to $20 \mathrm{~nm}[\mathrm{~J}]$. IEEE Transactions on Electron Devices, 2001, 47(12):2320-2325.

[9] Wong H S P, Chan K K, Taur Y. Self-aligned (top and bottom) double-gate MOSFET with a 25 $\mathrm{nm}$ thick silicon channel[J]. Electron Devices Meeting. iedm.technical Digest.international, 1998:427-430.

[10] Baccarani G, Reggiani S. A compact double-gate MOSFET model comprising quantummechanical and nonstatic effects[J]. Electron Devices IEEE Transactions on, 1999, 46(8):16561666.

[11] Yuan T. Analytical solution to a double-gate MOSFET with undoped body[J]. IEEE Electron Device Letters, 2000, 21(5):245-247.

[12] Tang S H, Chang L, Lindert N, et al. FinFET-a quasi-planar double-gate MOSFET[C]// SolidState Circuits Conference, 2001. Digest of Technical Papers. ISSCC. 2001 IEEE International. IEEE Xplore, 2001:118-119, 437.

[13] Rahman A, Lundstrom M S. A compact scattering model for the nanoscale double-gate MOSFET[J]. IEEE Transactions on Electron Devices, 2002, 62(3):481-489.

[14] Chan K K, Cohen G M, Taur Y, et al. Self-aligned double-gate MOSFET by selective epitaxy and silicon wafer bonding techniques: US, US6759710[P]. 2004.

[15] Kim J J, Roy K. Double gate-MOSFET subthreshold circuit for ultralow power applications[J]. IEEE Transactions on Electron Devices, 2004, 51(9):1468-1474. 
[16] Hanafi H I, Brown J J, Natzle W C. Damascene double-gate MOSFET with vertical channel regions: US, US $6835614 \mathrm{~B} 2[\mathrm{P}] .2004$.

[17] Tamersit K, Djeffal F, Meguellati M. Numerical Modeling of a Deep Submicron Gas Sensor Based on Double-Gate Graphene Nanoribbon Field-Effect Transistor[C]// The World Congress on Engineering. 2015.

[18] Owlia H, Keshavarzi P. Investigation of the novel attributes of a double-gate graphene nanoribbon FET with AlN high- $\kappa$ dielectrics[J]. Superlattices \& Microstructures, 2014, 75:613-620.

[19] Tamersit K, Djeffal F. Double-Gate Graphene Nanoribbon Field-Effect Transistor for DNA and Gas Sensing Applications: Simulation Study and Sensitivity Analysis[J]. IEEE Sensors Journal, 2016, 16(11):1-1.

[20] Dragoman M, Dinescu A, Dragoman D. Room temperature on-wafer ballistic graphene fieldeffect-transistor with oblique double-gate[J]. Journal of Applied Physics, 2016, 119(24):654-657. 